\title{
Efficient Method for the Synthesis of Diazaphospholidines: Toxicological Evaluation
}

\author{
Fouzia Bouchareb $^{1}$, Sihem Hessainia ${ }^{1}$, Malika Berredjem ${ }^{1, *}$ Hounaida Benbouzid ${ }^{2}$, Houria Djebbar, \\ Nour-Eddine Aouf ${ }^{1}$ \\ ${ }^{1}$ Laboratory of Applied Organic Chemistry, Badji-Mokhtar University BP12. Annaba, 23000, Algeria \\ ${ }^{2}$ Laboratory of Cell Toxicology, General Direction of Scientific Research and Technological Development, Algeria
}

\begin{abstract}
In this work, we described the synthesis of new heterocyclic organophosphorus compounds starting from a primary amine and phenyl phosphonic dichloride (PDCP). The introduction of a phosphoryl group into heterocyclic structures can generate potential chemical and biological activities. We have prepared the phosphoramidates in only one step. These compounds provide access to diazaphospholidine by intermolecular cyclization using dibromoethane. We chose primary amines (cyclohexylamine, benzylamine, propylamine and phenyl ethylamine) to prepare phosphoramidates. The toxicological activity of two synthesized molecules was evaluated.
\end{abstract}

Keywords Phenyl Phosphonic Dichloride, Phosphoramidate, Diazaphospholidine

\section{Introduction}

Organophosphorus compounds are ubiquitous in nature and find applications in the field of agriculture, medicine and industry. ${ }^{1,2}$ Some organophosphorus compounds have been described in the literature as inhibitors of bacterial $^{3}$, herbicides, insecticides, pesticides ${ }^{4}$, antifungal agents $^{5}$, anti-HIV ${ }^{6}$, anti-cancer ${ }^{7}$, antiviral and anti-infla mmatory ${ }^{8}$. An important group of this class is phosphoramidate, which have been used in many reactions and synthesis of organic compounds.

A number of research groups has become interested in organophosphorus heterocyclic compounds since they are finding extensive use as pesticides in agriculture, as stabilizers in polymers and as lubricant oil additives. An important family of this heterocycles is diazaphospholidine, which have been used in many reactions serve as ligands for transition metal complexes ${ }^{9}$ phosphorus ligands containing alternative donor units, such as phosphites ${ }^{10}$ or diazaphospholidines, ${ }^{11}$ have been less widely examined.

Herein, we report the synthesis of some novel diazaphospholidine derivatives (2a-d), and their corresponding precursors phosphoramidates (1a-d). choose to use phenylphosphonic dichloride to introduce phosphoramidate moiety. The toxicological activity of this compounds was evaluated.

* Corresponding author:

malika.berredjem@univ-annaba.org (Malika Berredjem)

Published online at http://journal.sapub.org/ajoc

Copyright (C) 2012 Scientific \& Academic Publishing. All Rights Reserved

\section{Results and discussion}

The phosphoramidates derivatives (1a-d), (Scheme 1) were prepared in a one-pot synthetic route, starting from corresponding primary amines with phenyl phosphonic dichloride in dry acetonitrile at $-5^{\circ} \mathrm{C}$. These compounds were obtained in good yields. The synthesis of diazaphosph-olidine was achieved by mixing 10 equivalents of dibromoetane with phosphoramidate in the presence of potassium carbonate. The heterocyclic compounds were obtained as a white solid in $67 \%$ yields.

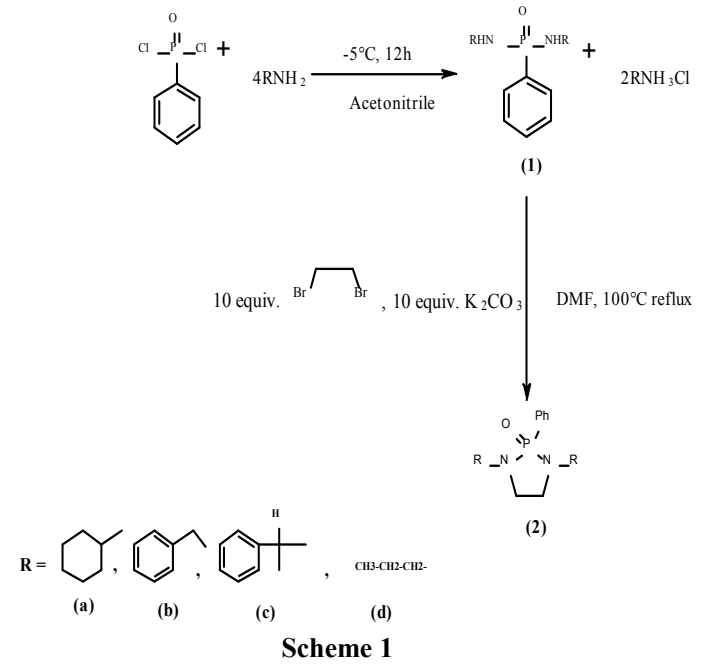

We describe the inhibitory effect of phosphoramidate on 
Paramecium Aurelia (1a) and (1b), the unicellular ciliate, Paramecium (Peniculia, Oligohymenophorea), is one of the most studied protists. Considerable research has been conducted on its morphology, physiology, genetics, nuclear reorganization, phylogeny, and the concept of 'species' [12].

these ciliates are excellent alternative models in toxicology. Our results showed a perturbation of respiratory metabolism, thus confirming the effect of these molecules on the respiratory chain of micro-organism.

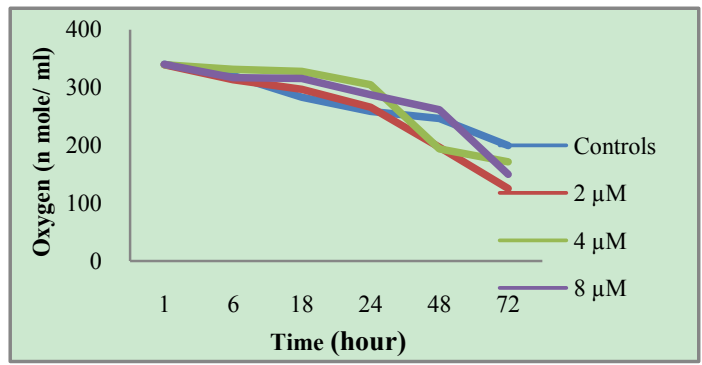

Figure 1. Effects of the molecule (1a) on the respiratory metabolism

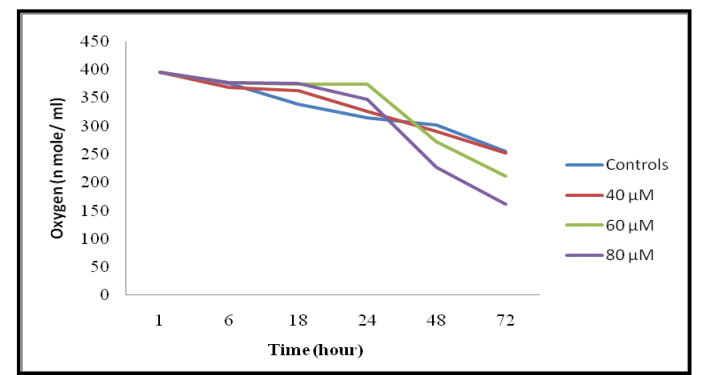

Figure 2. Effects of the molecule (1b) on the respiratory metabolism

The Figure 1 shows the effects of compound 1a on the paramecia metabolism respiratory at concentrations 2,4 and $8 \mu \mathrm{M}$. We note a slight inhibition of $\mathrm{O}_{2}$ consummation of paramecia treated with different concentrations of 1a reflecting a sharp slowdown of their respiratory activity between 6 and 24 hours. Beyond 24 hours, breathing of paramecia treated is strongly stimulated.

We note that the cells treated with different concentrations of $1 \mathrm{~b}$ present a sharp slowdown in their respiratory activity between 6 and 24 hours (figure 2). Beyond 24 hours, the respiration of paramecia treated is strongly stimulated.

\section{Experimental Section}

Melting points were determined in open capillary tubes on an Electro thermal apparatus and uncorrected. IR spectra were recorded on a perkin-Elmer FT-600 spectrometer. Proton nuclearmagnetic resonance was determined with a $360 \mathrm{WB}$ or AC $250-\mathrm{MHz}$ Bruker spectrometer using $\mathrm{CDCl}_{3}$ and DMSO- $\mathrm{d}_{6}$ as a solvent and TMS as an internal standard. Chemical shifts are reported in $\delta$ units (ppm). All coupling constants $(J)$ are reported in Hertz. Multiplicity is indicated as s (singlet), $\mathrm{d}$ (doublet), $\mathrm{t}$ (triplet), $\mathrm{m}$ (multiplet) and combination of these signals. Electron ionization mass spectra $(30 \mathrm{ev})$ were recorded in positive mode on a Water MicroMass ZQ. Hight-resolution mass were measured on a
Joel SX 102 mass spectrometer and recorded in FAB positive mode. All reactions were monitored by TLC on silica Merck h60 F254 (Art. 5554) precoated alumium plates and were developed by spraying with ninhydrin solution.

\subsection{Synthesis of Phenyl Phosphoramidates}

To a stirred solution of phenyl phosphonic dichloride $(0.71 \mathrm{~mL}, 5 \mathrm{mmol})$ in dry acetonitrile $(35 \mathrm{~mL})$, a solution of primary amine (1.98 g, $20 \mathrm{mmol}$, 4 equiv) was added dropwise at $-5^{\circ} \mathrm{C}$. After $12 \mathrm{~h}$, the solvent was evaporated under vacuum and the oily product was washed with distilled water. The organic layer was dried over sodium sulfate and concentrated. The products were purified by column chromatography on silica gel $\left(\mathrm{CH}_{2} \mathrm{Cl}_{2} / \mathrm{MeOH} 9 / 1\right)$. The phosphoramidates were obtained as a white solid in excellent yields.

Bis (cyclohexylamino) phenylphosphine oxide 1a: Yield: $89 \% . \mathrm{M}=320 \mathrm{~g} / \mathrm{mol}\left[\mathrm{C}_{18} \mathrm{H}_{29} \mathrm{~N}_{2} \mathrm{OP}\right] . \mathrm{Mp} .156-158^{\circ} \mathrm{C} . \mathrm{R}_{\mathrm{f}}$ $=0.625\left(\mathrm{CH}_{2} \mathrm{Cl}_{2} / \mathrm{MeOH}\right)$. IR $\left(\mathrm{KBr}, \mathrm{cm}^{-1}\right): 3155.3 \mathrm{~cm}^{-1}(\mathrm{NH})$, $1461.9 \mathrm{~cm}^{-1}\left(\mathrm{C}=\mathrm{C}_{\mathrm{Ar}}\right), 1180.4 \mathrm{~cm}^{-1} \quad(\mathrm{P}=\mathrm{O}), 1110.9 \mathrm{~cm}^{-1}$ (C-N-P). ${ }^{1} \mathrm{H}$ NMR $\left(\mathrm{CDCl}_{3}, 250 \mathrm{MHz}\right): 7.90$ (m, 2H, H-Ar); 7.50 (m, 3H, H-Ar); 3.15 (m, 2H, CH-cyc); 2.40 (d, $J=9.3$ $\mathrm{Hz}, 2 \mathrm{H}, \mathrm{NH}-\mathrm{P}=\mathrm{O}) ; 1.90\left(\mathrm{~m}, 4 \mathrm{H}, \mathrm{CH}_{2}\right.$-cyc); $1.65(\mathrm{~m}, 4 \mathrm{H}$, $\mathrm{CH}_{2}$-cyc); 1.15 (m, $12 \mathrm{H}, \mathrm{CH}_{2}$ cycl). ${ }^{31} \mathrm{P}$ NMR $\left(\mathrm{CDCl}_{3}\right.$, $200.7 \mathrm{MHz}) \delta=16.42 \mathrm{ppm} .{ }^{13} \mathrm{C} \mathrm{NMR}\left(\mathrm{CDCl}_{3}, 250 \mathrm{MHz}\right)$ : 134 (CH-Ar); 132 (CH-Ar); 129 (CH-Ar); 55 (CH-Hex); $35\left(\mathrm{CH}_{2}-\mathrm{Hex}\right) ; 25\left(\mathrm{CH}_{2}-\mathrm{Hex}\right) ; 24.8\left(\mathrm{CH}_{2}-\mathrm{Hex}\right)$.

Bis (benzylamino) phenylphosphine oxide 1b: Yield: $91 \% . \mathrm{M}=336 \mathrm{~g} / \mathrm{mol}\left[\mathrm{C}_{20} \mathrm{H}_{21} \mathrm{~N}_{2} \mathrm{OP}\right] . \mathrm{Mp} .159-161^{\circ} \mathrm{C} . \mathrm{R}_{\mathrm{f}}=$ $0.60 \quad\left(\mathrm{CH}_{2} \mathrm{Cl}_{2} / \mathrm{MeOH}\right)$. IR $\left(\mathrm{KBr}, \quad \mathrm{cm}^{-1}\right): 3163 \mathrm{~cm}^{-1}$ $(\mathrm{NH}), 1461.9 \mathrm{~cm}^{-1} \quad\left(\mathrm{C}=\mathrm{C}_{\mathrm{Ar}}\right), 1253.6 \mathrm{~cm}^{-1} \quad(\mathrm{P}=\mathrm{O}), 1118.6$ $\mathrm{cm}^{-1}$ (C-N-P). ${ }^{1} \mathrm{H}$ NMR $\left(\mathrm{CDCl}_{3}, 250 \mathrm{MHz}\right): 7.9(\mathrm{~m}, 2 \mathrm{H}$, H-Ar); 7.5 (m, 3H, H-Ar); 7.25 (m, 10H, H-Ar); 4.15 (d, $J=$ $\left.6.85 \mathrm{~Hz}, 4 \mathrm{H}, \mathrm{CH}_{2}-\mathrm{N}\right) ; 3.0(\mathrm{~m}, 2 \mathrm{H}, \mathrm{NH}-\mathrm{P}=\mathrm{O}) .{ }^{31} \mathrm{P}$ NMR $\left(\mathrm{CDCl}_{3}, 200.7 \mathrm{MHz}\right) \delta=16.33 \mathrm{ppm} .{ }^{13} \mathrm{C}$ NMR $\left(\mathrm{CDCl}_{3}\right.$, 250MHz): 141 (C-Ar); 134.1 (C-Ar); 134 (CH-Ar); 132 (CH-Ar); 129 (CH-Ar); 128.5 (CH-Ar); 127 (CH_Ar); 126.6 (CH-Ar); $44\left(\mathrm{CH}_{2}\right)$.

Bis (phenylethylamino) phenylphosphine oxide 1c: Yield: $87.15 \% . \mathrm{M}=364 \mathrm{~g} / \mathrm{mol}\left[\mathrm{C}_{22} \mathrm{H}_{25} \mathrm{~N}_{2} \mathrm{OP}\right] . \mathrm{Mp} .164-167{ }^{\circ} \mathrm{C}$. $\mathrm{R}_{\mathrm{f}}=0.63\left(\mathrm{CH}_{2} \mathrm{Cl}_{2} / \mathrm{MeOH}\right) \cdot \mathrm{IR}\left(\mathrm{KBr}, \mathrm{cm}^{1}\right): 3209.3 \mathrm{~cm}^{-1}$ $(\mathrm{NH}), 1450.4 \mathrm{~cm}^{-1}\left(\mathrm{C}=\mathrm{C}_{\mathrm{Ar}}\right), 1191.9 \mathrm{~cm}^{-1}(\mathrm{P}=\mathrm{O}), 1029.9$ $\mathrm{cm}^{-1}$ (C-N-P). ${ }^{1} \mathrm{H}$ NMR $\left(\mathrm{CDCl}_{3}, 250 \mathrm{MHz}\right): 7.75$ (m, 2H, $\mathrm{H}-\mathrm{Ar}$ ); 7.4 (m, 3H, H-Ar); 7.35-7 (m, 10H, H-Ar); 4.60 (m, $\left.1 \mathrm{H},{ }^{*} \mathrm{CH}-\mathrm{NH}\right) ; 4.35\left(\mathrm{~m}, \quad 1 \mathrm{H},{ }^{*} \mathrm{CH}-\mathrm{NH}\right) ; 2.75 \quad(\mathrm{~s}, \quad 2 \mathrm{H}$, NH-P $=0) ; 1.5\left(\mathrm{~d}, J=6.25 \mathrm{~Hz}, 3 \mathrm{H}, \mathrm{CH}_{3}\right) ; 1.3(\mathrm{~d}, J=6.77 \mathrm{~Hz}$, $\left.3 \mathrm{H}, \mathrm{CH}_{3}\right) .{ }^{31} \mathrm{P} \mathrm{NMR}\left(\mathrm{CDCl}_{3}, 200.7 \mathrm{MHz}\right) \delta=16.65 \mathrm{ppm}$. ${ }^{13} \mathrm{C}$ NMR $\left(\mathrm{CDCl}_{3}, 250 \mathrm{MHz}\right): 145$ (C-Ar); 134.2 (C-Ar); 132.1 (CH-Ar); 130 (2 C, CH-Ar); 128.8 (CH-Ar); 128.5 (CH-Ar); 126.9 (CH-Ar); 126.7 (CH_Ar); 55 (CH); 24 $\left(\mathrm{CH}_{3}\right)$.

Bis (propylamino) phenylphosphine oxide 1d: Yield: $88.53 \% . \mathrm{M}=240 \mathrm{~g} / \mathrm{mol}\left[\mathrm{C}_{12} \mathrm{H}_{21} \mathrm{~N}_{2} \mathrm{OP}\right] . \mathrm{Mp} .150-153{ }^{\circ} \mathrm{C}$. $\mathrm{R}_{\mathrm{f}}=0.617\left(\mathrm{CH}_{2} \mathrm{Cl}_{2} / \mathrm{MeOH}\right)$. IR $\left(\mathrm{KBr}, \mathrm{cm}^{-1}\right): 3193.9 \mathrm{~cm}^{-1}$ (NH), $1465.8 \mathrm{~cm}^{-1}(\mathrm{C}=\mathrm{C}$ Ar $), 1200 \mathrm{~cm}^{-1}(\mathrm{P}=\mathrm{O}), 1033.8 \mathrm{~cm}^{-1}$ (C-N-P). ${ }^{1} \mathrm{H}$ NMR ( $\left.\mathrm{CDCl}_{3}, 250 \mathrm{MHz}\right): 7.9$ (m, 2H, H-Ar); 
7.5 (m, 3H, H-Ar); 2.9 (m, 4H, $\left.\mathrm{CH}_{2}-\mathrm{NH}\right) ; 2.5(\mathrm{~m}, 2 \mathrm{H}$, $\mathrm{NH}-\mathrm{P}=\mathrm{O}) ; 1.5\left(\mathrm{~m}, 4 \mathrm{H}, \mathrm{CH}_{2}-\mathrm{CH}_{3}\right) ; 0.9$ (t, $J_{I}=7.31 \mathrm{~Hz}, J_{2}=$ $\left.7.46 \mathrm{~Hz}, 6 \mathrm{H}, \mathrm{CH}_{3}\right) .{ }^{31} \mathrm{P} \mathrm{NMR}\left(\mathrm{CDCl}_{3}, 200.7 \mathrm{MHz}\right) \delta=16.4$ ppm. ${ }^{13} \mathrm{C}$ NMR $\left(\mathrm{CDCl}_{3}, 250 \mathrm{MHz}\right): 135$ (C-Ar); 131.5 (CH-Ar); 128.3 ( CH-Ar); 128.30 (CH-Ar); $42.5\left(\mathrm{CH}_{2-} \mathrm{NH}\right)$; $25.3\left(\mathrm{CH}_{2}-\mathrm{CH}_{3}\right) ; 11.3\left(\mathrm{CH}_{3}\right)$.

\subsection{Synthesis of Diazaphospholidine}

A solution of phenyl phosphoramidates $(1 \mathrm{~g}, 3 \mathrm{mmol}, 1$ equiv) and excess of $\mathrm{K}_{2} \mathrm{CO}_{3}$ (4.14 g, $30 \mathrm{mmol}, 10$ equiv) in DMF (2 mL) was stirred, a solution of 1, 2- dibromoethane ( $2.59 \mathrm{~g}, 30 \mathrm{mmol}, 10$ equiv) was added dropwise. The reaction mixture was refluxed for $2 \mathrm{~h}$. The residue was evaporated in vacuum. The products were purified by column chromatography on silica gel $\left(\mathrm{CH}_{2} \mathrm{Cl}_{2} / \mathrm{MeOH} 9 / 1\right)$ afforded diazaphospholidines as a white solid in $67 \%$ yields.

1,3-cyclohexyl, 2-phenyl diazaphospholidin-2-oxide 2a: Yield: $66.78 \%$. $M=346 \mathrm{~g} / \mathrm{mol}\left[\mathrm{C}_{20} \mathrm{H}_{31} \mathrm{~N}_{2} \mathrm{OP}\right] . \quad \mathrm{Mp}$. $171-173^{\circ} \mathrm{C} . \mathrm{R}_{\mathrm{f}}=0.83\left(\mathrm{CH}_{2} \mathrm{Cl}_{2} / \mathrm{MeOH}\right)$. IR $\left(\mathrm{KBr}, \mathrm{cm}^{-1}\right)$ : $1451.5 \mathrm{~cm}^{-1}\left(\mathrm{C}=\mathrm{C}_{\mathrm{Ar}}\right), 1181.2 \mathrm{~cm}^{-1}(\mathrm{P}=\mathrm{O}), 1109.4 \mathrm{~cm}^{-1}$ (C-N-P). ${ }^{1} \mathrm{H}$ NMR ( $\left.\mathrm{CDCl}_{3}, 250 \mathrm{MHz}\right): 7.95$ (m, 2H, H-Ar); 7.55 (m, 3H, H-Ar); 3.20 (m, 2H, CH-cyc); 2.5 (t, $J_{I}=9.4$ $\mathrm{Hz}, J_{2}=9.42 \mathrm{~Hz}, 4 \mathrm{H}, \mathrm{CH}_{2}$-cyc à 5$) ; 1.95(\mathrm{~m}, 4 \mathrm{H}$, $\mathrm{CH}_{2}$-cyc);1.70 (m, 4H, $\mathrm{CH}_{2}$-cyc); 1.20 (m, $12 \mathrm{H}, \mathrm{CH}_{2}$-cycl). ${ }^{31} \mathrm{P}$ NMR $\left(\mathrm{CDCl}_{3}, 200.7 \mathrm{MHz}\right) \delta=33.55 \mathrm{ppm} .{ }^{13} \mathrm{C}$ NMR $\left(\mathrm{CDCl}_{3}, 250 \mathrm{MHz}\right)$ : 134.2 (C-Ar); 131.9 (1 C, CH-Ar); 130.3 (CH-Ar); 128.8 (CH-Ar); 60 (CH-Hex); 44.1 $\left(\mathrm{CH}_{2}\right.$-cyc $) ; 33.1\left(\mathrm{CH}_{2}-\mathrm{Hex}\right) ; 25.7\left(\mathrm{CH}_{2}-\mathrm{Hex}\right) ; 25.1\left(\mathrm{CH}_{2}-\right.$ Hex).

1, 3-benzyl, 2-phenyl diazaphospholidin-2-oxide $\mathbf{2 b}$ : Yield: $69.42 \% . \quad M=346 \mathrm{~g} / \mathrm{mol} \quad\left[\mathrm{C}_{22} \mathrm{H}_{31} \mathrm{~N}_{2} \mathrm{OP}\right] . \quad$ Mp. 174-177 ${ }^{\circ} \mathrm{C} . \mathrm{R}_{\mathrm{f}}=0.81\left(\mathrm{CH}_{2} \mathrm{Cl}_{2} / \mathrm{MeOH}\right)$. IR $\left(\mathrm{KBr}, \mathrm{cm}^{-1}\right)$ : $1460.1 \mathrm{~cm}^{-1} \quad\left(\mathrm{C}=\mathrm{C}_{\mathrm{Ar}}\right), 1253.4 \mathrm{~cm}^{-1}(\mathrm{P}=\mathrm{O}), \quad 1117.6 \mathrm{~cm}^{-1}$ (C-N-P). ${ }^{1} \mathrm{H}$ NMR $\left(\mathrm{CDCl}_{3}, 250 \mathrm{MHz}\right): 7.95$ (m, 2H, H-Ar); 7.55 (m, 3H, H-Ar); 7.30 (m, 10H, H-Ar); 4.20 (d, $J=6.95$ $\left.\mathrm{Hz}, 4 \mathrm{H}, \mathrm{CH}_{2}-\mathrm{N}\right) ; 3.0\left(\mathrm{t}, J_{I}=8.8 \mathrm{~Hz}, J_{2}=8.9 \mathrm{~Hz}, 4 \mathrm{H}, \mathrm{CH}_{2} \mathrm{cyc}\right.$ à 5). ${ }^{31} \mathrm{P} \mathrm{NMR}\left(\mathrm{CDCl}_{3}, 200.7 \mathrm{MHz}\right) \delta=33.34 \mathrm{ppm}$.

${ }^{13} \mathrm{C} \mathrm{NMR}\left(\mathrm{CDCl}_{3}, 250 \mathrm{MHz}\right): 136.4$ (C-Ar); $134.2(\mathrm{C}-\mathrm{Ar})$; 134.1 (CH-Ar); 132.3 (CH- Ar); 128.8 (CH-Ar); 128.5 (CH-Ar); 127.9 (CH_Ar); 127(CH-Ar); $73.3\left(\mathrm{CH}_{2}\right) ; 45.6$ $\left(\mathrm{CH}_{2}\right)$.

1, 3-phenylethyl, 2-phenyl, diazaphospholidin-2-oxide 2c: Yield: $64.29 \%$. $\quad \mathrm{M}=390 \mathrm{~g} / \mathrm{mol} \quad\left[\mathrm{C}_{24} \mathrm{H}_{27} \mathrm{~N}_{2} \mathrm{OP}\right] . \quad$ Mp. $178-179^{\circ} \mathrm{C} . \quad \mathrm{R}_{\mathrm{f}}=0.80\left(\mathrm{CH}_{2} \mathrm{Cl}_{2} / \mathrm{MeOH}\right)$. IR $\left(\mathrm{KBr}, \mathrm{cm}^{-1}\right)$ : $1458.1 \mathrm{~cm}^{-1}(\mathrm{C}=\mathrm{C}$ Ar $), 1184.2 \mathrm{~cm}^{-1}(\mathrm{P}=\mathrm{O}), 1107.1 \mathrm{~cm}^{-1}$ (C-N-P). ${ }^{1} \mathrm{H}$ NMR $\left(\mathrm{CDCl}_{3}, 250 \mathrm{MHz}\right): 7.75$ (m, 2H, H-Ar); 7.5 (m, 3H, H-Ar); 7.4-7.0 (m, 10H, H-Ar); $4.70(\mathrm{~m}$, $\left.1 \mathrm{H},{ }^{*} \mathrm{CH}-\mathrm{NH}\right) ; 4.40\left(\mathrm{~m}, 1 \mathrm{H},{ }^{*} \mathrm{CH}-\mathrm{NH}\right) ; 2.8\left(\mathrm{t}, J_{I}=9.34 \mathrm{~Hz}\right.$, $J_{2}=9.48 \mathrm{~Hz}, 2 \mathrm{H}, \mathrm{CH}_{2}$-cyc à 5$) ; 2.7\left(\mathrm{t}, J_{I}=8.82 \mathrm{~Hz}, J_{2}=8.57\right.$ $\mathrm{Hz}, 2 \mathrm{H}, \mathrm{CH}_{2}$-cyc à 5); $1.45\left(\mathrm{~d}, J=6 \mathrm{~Hz}, 3 \mathrm{H}, \mathrm{CH}_{3}\right) ; 1.30(\mathrm{~d}$, $\left.J=6.76 \mathrm{~Hz}, 3 \mathrm{H}, \mathrm{CH}_{3}\right) \cdot{ }^{31} \mathrm{P}$ NMR $\left(\mathrm{CDCl}_{3}, 200.7 \mathrm{MHz}\right) \delta=$ 34.64 ppm. ${ }^{13} \mathrm{C}$ NMR $\left(\mathrm{CDCl}_{3}, 250 \mathrm{MHz}\right): 138.3$ (C-Ar); 134.2 (C-Ar); 134.1 (CH-Ar); 132.3 (CH-Ar); 128.8 (CH-Ar); 128.5 (CH-Ar); 127.9 (CH-Ar); 127 (CH_Ar); $45.6(\mathrm{CH}) ; 43.4\left(\mathrm{CH}_{2}\right) ; 20.8\left(\mathrm{CH}_{3}\right)$.
1, 3-propyl, 2-phenyl diazaphospholidin-2-oxide 2d: Yield: $68.31 \% . \mathrm{M}=266 \mathrm{~g} / \mathrm{mol}\left[\mathrm{C}_{14} \mathrm{H}_{23} \mathrm{~N}_{2} \mathrm{OP}\right] . \mathrm{Mp}$. $168-170^{\circ} \mathrm{C} . \mathrm{R}_{\mathrm{f}}=0.82\left(\mathrm{CH}_{2} \mathrm{Cl}_{2} / \mathrm{MeOH}\right) . \quad \mathrm{IR}\left(\mathrm{KBr}, \mathrm{cm}^{-1}\right)$ : $1451.4 \mathrm{~cm}^{-1}(\mathrm{C}=\mathrm{C}$ Ar $), 1192.9 \mathrm{~cm}^{-1} \quad(\mathrm{P}=\mathrm{O}), 1029.3 \mathrm{~cm}^{-1}$ (C-N-P). ${ }^{1} \mathrm{H}$ NMR $\left(\mathrm{CDCl}_{3}, 250 \mathrm{MHz}\right): 7.80$ (m, 2H, H-Ar); $7.65(\mathrm{~m}, 3 \mathrm{H}, \mathrm{H}-\mathrm{Ar}) ; 3.0\left(\mathrm{~m}, 4 \mathrm{H}, \mathrm{N}-\mathrm{CH}_{2}-\mathrm{CH}_{2}\right) ; 2.5\left(\mathrm{t}, J_{I}=\right.$ $9.37 \mathrm{~Hz}, J_{2}=9.43 \mathrm{~Hz}, 4 \mathrm{H}, \mathrm{CH}_{2}$ cyc à 5$) ; 1.55(\mathrm{~m}, 4 \mathrm{H}$, $\left.-\mathrm{CH}_{2}-\mathrm{CH}_{3}\right) ; 0.95\left(\mathrm{t}, J_{I}=9.35 \mathrm{~Hz}, J_{2}=9.49 \mathrm{~Hz}, 6 \mathrm{H}, \mathrm{CH}_{3}\right) .{ }^{31} \mathrm{P}$ NMR $\left(\mathrm{CDCl}_{3}, 200.7 \mathrm{MHz}\right) \delta=33.7 \mathrm{ppm} .{ }^{13} \mathrm{C} \mathrm{NMR}\left(\mathrm{CDCl}_{3}\right.$, 250MHz): 134.6 (C-Ar); 131.5 (CH-Ar); 128.3 (CH-Ar); $128.3(\mathrm{CH}-\mathrm{Ar}) ; 77\left(\mathrm{CH}_{2} \mathrm{NH}\right) ; 42.57\left(\mathrm{CH}_{2}\right) ; 25.29\left(\mathrm{CH}_{2}-\right)$; $11.28\left(\mathrm{CH}_{3}\right)$.

\section{Conclusions}

This work is based essentially on the synthesis of a new family of modified diazaphospholidines. We achieved the synthesis of phenyl phosphoramidates derived from primary amines (cyclohexylamine, benzylamine, propylamine, and ethylphenylamine) with phenyl phosphonic dichloride in one step. Our toxicological results showed a perturbation of respiratory metabolism, confirming the effect of these molecules on the respiratory chain. Thus we can conclude that the xenobiotics tested are cytotoxic. This toxicity is manifested by the loss of linearity of the trajectory and mobility followed by an inhibition of cell growth.

\section{ACKNOWLEDGMENTS}

This work was generously supported by the (Direction Generale de la Recherche Scientifique et du Développement Technologique, DGRS-DT), Algerian Ministry of Scientific Research, (FNR) and CMEP 08 MDU 729.

\section{REFERENCES}

[1] E. Breuer, The Chemistry of Organophosphorus Compounds, Heartly, F. R. Ed., John Wiley and Sons, New York, 1996, Vol 4, 653

[2] Prakasha, T. K., Day O. R., Holmes R. R., 1994, J. Am. Chem. Soc., 116, 8095

[3] (a) Faraci, W. S., Yang, B. V., D. O'Rourke, R. W. Spencer., 1995, Bioorg. Med. Chem., 3, 605. (b) Bhatia, M. S. Jit, P., 1976, Experientia, 32, 1111.(c) Alexakis, A.; Mutti, S.; Normant, J. F. J.; 1991, Am. Chem. Soc. 113, 6332. (d) Feringa, B. L., de Vries, A. H., Meetsma. A., 1996, Angew. Chem. Int. Ed., 35, 2374

[4] (a) C. Fest, K. J. Schmidt, The Chemistry of Organophosphorus Pesticides Springer-Verlag, Berlin, 1982, 12. (b) Nivsarkar, M. Gupta, A. K. Kaushik, M. P., 2004, Tetrahedron Lett. 45, 6863

[5] Ali, H. M., Ali,M. K., 2000, Bull.Environ. Contam. Toxicol. 
65,415

[6] Mehellou, Y., MeGuigan, C., Brancale, A., Balzarini, J., 2007, Bioor. Med. Chem. Lett. 17, 3666.

[7] Guigan, C. M., Thiery, J. C., Daverio, F., Jiang, W. J., Davies, G., Mason, M., 2005, Bioorg. Med.Chem. 13, 3219

[8] Wittine, K., Benci, K., Rajic, Z., Zorc, B., Kralj, M., Marjanovic, M., Pavelic, K., De Clercq, E., Andrei, G., Snoeck, R., Balzarini, J., Mintas, M., 2009, Eur. J. Med. Chem. 44,143

[9] a) Alexakis, A., Mutti, S., Normant, J.F.J., 1991, Am. Chem. Soc. 113, 6332. (b) Feringa, B. L., de Vries, A. H., Meetsma, A., 1996, Angew. Chem. Int. Ed. 35, 2374
[10] (a) Horiuchi, T., Ohta, T., Shirakawa, E., Nozaki, K., Takaya, H., 1997, J. Org. Chem. 62, 4285. (b) Nozaki, K., Itoi, Y., Shibahara, F., Shirakawa, E., Ohta, K., Takaya, H., Hiyama, T. J., 1998, Am. Chem. Soc. 120, 4051. (c) Nozaki, K., Sato, N., Tonomura, Y., Yasutomi, M.,Takaya, H., Hiyama, T., Matsubara, T., Koga, N. J., 1997, Am. Chem. Soc. 119, 12779. (d) Feringa, B. L., Pineschi, M., Arnold, L. A., Imbos, R., 1997, Angew. Chem. Int. Ed. Engl. 36, 2620

[11] (a) Longeau, A., Durand, S., Spiegel, A., Knochel, P., 1997 Tetrahedron: Asymmetry 8, 987. (b) Vasconcelos, I. C. F., Anderson, G. K., Rath, N. P., Spilling, C. D., 1998, Tetrahedron Lett. 9, 927

[12] Ali, H. M., Ali, M. K., 2000, Bull.Environ. Contam. Toxicol. 65,415 